\title{
SOLUTIONS FOR THE LINEAR QUADRATIC CONTROL PROBLEM OF MARKOV J UMP LINEAR SYSTEMS
}

\author{
J.B.R. do Val ${ }^{1}$, J.C. Geromel ${ }^{1}$ and O.L.V. Costa ${ }^{2}$
}

\begin{abstract}
The paper is concerned with recursive methods for obtaining the stabilizing solution of coupled algebraic Riccati equations arising in the linear quadratic control for Markovian jump linear systems, by solving at each iteration uncoupled algebraic Riccati equations. It is shown that the new updates carried out at each iteration represent approximations of the original control problem by control problems with receding horizon, for which some sequences of stopping times define the terminal time. Under this approach it follows that, unlike previous results, no initialization conditions are required to guarantee the convergence of the algorithms. The methods can be ordered in terms of number of iterations to reach convergence, and comparisons with existing methods in the current literature are also presented. Moreover, we also extend and generalize current results in the literature for the existence of the mean square stabilizing solution of the coupled algebraic Riccati equations.
\end{abstract}

Keywords: Linear Quadratic Control, Markov Jump Linear Systems, Nonstandard Riccati equation, Stopping Time Problems.

${ }^{1}$ LAC-DT, Faculty of Electrical Engineering, UNICAMP, C.P. 6101, 13081-970 - Campinas - SP Brazil. Correspondence should be sent to the first author, e-mail address: jbosco@dt.fee.unicamp.br

${ }^{2}$ University of São Paulo, Department of Electronic Engineering, 05508-900 - São Paulo - SP - Brazil. 


\section{Introduction}

In this paper we consider the linear quadratic (LQ) control problem for Markov Jump Linear Systems (MJLS) with linear system state and Markov chain state observations. In this formulation, the mode of the linear system changes according to an underlying Markov chain, and the control has complete access to the state variables. The MJLS comprise an important class of stochastic time-variant systems that have gained much evidence due to, among other things, the ability of modeling random abrupt changes occurring in a linear plant structure. Several results can be found nowadays in the current literature concerning stability and control, e.g. [1]-[4],[6],[8]-[11],[13]. The concept of mean-square stability is the appropriate one for MJLS, and it has provided the analytical grounds for the study of control of these systems. Using this concept, it has been shown that the solution of the LQ-optimal control problem for MJLS can be given in terms of the solution of an interconnected set of algebraic Riccati equations (ISARE), c.f. [1],[2],[9],[11].

The main result of this paper concerns the problem of obtaining the solution for the ISARE numerically. Methods for solving the ISARE that employ recursions on some set of uncoupled standard algebraic Riccati equations, or uncoupled standard Lyapunov equations can be found in the literature, see [1] and [9]. Also, in a different approach, a LMI formulation [2] is encountered. We present in this paper a new approach for solving the ISARE using recursions of uncoupled standard algebraic Riccati equations, which generalizes and unifies previous results proved in [1]. In particular the approach adopted here shows that no initialization condition is required to guarantee convergence of the algorithms.

The novelty of our approach is based on using dynamic programming arguments applied to a sequence of finite horizon problems with receding horizon. We present two algorithms based on iterations of uncoupled algebraic Riccati equations and show how each of these algorithms are associated with truncated quadratic optimal control problems with different sequences of stopping times for defining the terminal time. As a byproduct, the analysis eliminates any requirement on the initialization of the Riccati iteration methods for convergence. Previous results state restrictions on the initial set of matrices, and new updates are only considered after the end of a cycle, see [1]. We also set up a link between the convergence of these methods and the mean square stabilizability of a MJLS, see Theorem 3.3.

We compare the methods analytically and show how they can be ordered in terms of the rate of convergence, see Theorem 3.2. Under some initialization conditions we can also show the Riccati methods will converge faster than the Lyapunov method. This apparent advantage of the former method needs confirmation, since it is important to acknowledge that the computational effort to solve a standard Riccati equation is larger than that to solve a comparable Lyapunov equation. Thus, it is not clear beforehand which method will attain the best performance in terms of the overall time for convergence. An outcome favorable to the Riccati method is verified in some examples in section 4.

In section 4, we also compare numerically these solutions with the convex method applied to the solution of the problem for MJLS. For the control problem with complete state observation and perfectly known parameters, as set here or in [2], we confirm that a more efficient technique is provided by the recursive methods proposed here.

We also extend and generalize some current conditions for the existence of the mean square stabilizing solution for the coupled algebraic Riccati equations. By using the results established in [7], we can replace the observability condition derived in [11] (see Proposition 2.2) by the concept of mean square detectability (see Definition 2.1 and Theorem 2.1) or by the observability of each mode of the system with real part greater than a certain value (see Theorem 2.2).

The next section presents the control problem and some preliminary results concerning new conditions for the existence of the mean square stabilizing solution of the coupled algebraic Riccati equations, and some properties of the stochastic system and the associated algebraic Riccati and Lyapunov equations employed by the iterative methods. The main results appear in section 3 in the form 
of two companion theorems, Theorems 3.1 and 3.2. Numerical examples are presented in section 4 to expose the results and the points discussed above, and further conclusions are collected in section 5 .

\section{Basic Definitions and Preliminary Results}

\subsection{Basic Definitions}

We consider here the following interconnected set of algebraic Riccati equations (ISARE) in $X_{i}$, $i=1, \ldots, N$,

$$
A_{i}^{\prime} X_{i}+X_{i} A_{i}+\mathcal{E}_{i}(X)+C_{i}^{\prime} C_{i}-\left(X_{i} B_{i}+C_{i}^{\prime} D_{i}\right)\left(D_{i}^{\prime} D_{i}\right)^{-1}\left(B_{i}^{\prime} X_{i}+D_{i}^{\prime} C_{i}\right)=0, \quad i=1, \ldots, N
$$

where we set

$$
\mathcal{E}_{i}(X):=\sum_{j=1}^{N} \lambda_{i j} X_{j}
$$

and we assume that $D_{i}^{\prime} D_{i}>0$, for each $i=1, \ldots, N$. These equations arise when one considers the problem of minimizing the functional

$$
J\left(x_{0}, \theta_{0}\right)=\min E\left\{\int_{0}^{\infty}\left\|C_{\theta(t)} x(t)+D_{\theta(t)} u(t)\right\|^{2}\right\} d t
$$

where, in a probabilistic space $\left(\Omega, \Im,\left\{\Im_{t}\right\}, \mathbb{P}\right)$, the minimization is over $\{u(t) ; t \geq 0\}$ and each $u(t)$ is $\Im_{t}$ measurable. The jump and state variables $\{(\theta(t), x(t)) ; t \geq 0\}$ should satisfy

$$
\dot{x}(t)=A_{\theta(t)} x(t)+B_{\theta(t)} u(t), \quad x(0)=x, \theta(0)=\theta_{0}
$$

and $\theta(t)$ is a continuous time Markov chain taking values in $\{1, \ldots, N\}$ with transition rate matrix $\Lambda=\left[\lambda_{i j}\right], i, j=1, \ldots, N$. This process belongs to the class of strong Markov processes, c.f. [5]. We use the notation $\mathcal{A}=\left(A_{1}, \ldots, A_{N}\right), \mathcal{B}=\left(B_{1}, \ldots, B_{N}\right), \mathcal{C}=\left(C_{1}, \ldots, C_{N}\right)$, and we introduce also $\bar{A}_{i}=$ $A_{i}-B_{i}\left(D_{i}^{\prime} D_{i}\right)^{-1} D_{i}^{\prime} C_{i}, \bar{C}_{i}=\left(I-D_{i}\left(D_{i}^{\prime} D_{i}\right)^{-1} D_{i}^{\prime}\right) C_{i}$, which form the correspondent collections $\overline{\mathcal{A}}=$ $\left(\bar{A}_{1}, \ldots, \bar{A}_{N}\right)$ and $\overline{\mathcal{C}}=\left(\bar{C}_{1}, \ldots, \bar{C}_{N}\right)$. We need the following definitions and results.

Definition 2.1: We say that $(\mathcal{A}, \mathcal{B})$ is mean square stabilizable if we can find $\mathcal{K}=\left(K_{1}, \ldots, K_{N}\right)$ such that $E\left(\|x(t)\|^{2}\right) \rightarrow 0$ as $t \rightarrow \infty$, where $\dot{x}(t)=\left(A_{\theta(t)}+B_{\theta(t)} K_{\theta(t)}\right) x(t)$ for any initial conditions $x(0)$ and $\theta(0)$. In this case we say that $\mathcal{K}$ stabilizes $(\mathcal{A}, \mathcal{B})$. Similarly we say that $(\overline{\mathcal{C}}, \overline{\mathcal{A}})$ is mean square detectable if we can find $\mathcal{H}=\left(H_{1}, \ldots, H_{N}\right)$ such that $E\left(\|x(t)\|^{2}\right) \rightarrow 0 \quad$ as $t \rightarrow \infty$ where $\dot{x}(t)=\left(\bar{A}_{\theta(t)}+H_{\theta(t)} \bar{C}_{\theta(t)}\right) x(t)$ for any initial conditions $x(0)$ and $\theta(0)$.

Definition 2.2: We say that $\mathcal{P}=\left(P_{1}, \ldots, P_{N}\right)$ is a positive definite (semi-definite respectively) solution of (2.1) if $P_{i}>0\left(P_{i} \geq 0\right)$ and satisfies $(2.1)$ for $i=1, \ldots, N$. We say that $\mathcal{P}$ is the mean square stabilizing solution of (2.1) if it satisfies (2.1) and $\mathcal{K}=\left(K_{1}, \ldots, K_{N}\right)$ with

$$
K_{i}=-\left(D_{i}^{\prime} D_{i}\right)^{-1}\left(B_{i}^{\prime} P_{i}+D_{i}^{\prime} C_{i}\right)
$$

stabilizes $(\mathcal{A}, \mathcal{B})$ in the mean square sense.

The following result can be found in [8], [11] and [13]. 
Proposition 2.1: The following assertions are equivalent:

a) $(\mathcal{A}, \mathcal{B})$ is mean square stabilizable;

b) For some $\mathcal{K}=\left(K_{1}, \ldots, K_{N}\right)$, there exists $\mathcal{M}=\left(M_{1}, \ldots, M_{N}\right), M_{i}>0$ for $i=1, \ldots, N$, such that

$$
\left(A_{i}+B_{i} K_{i}\right)^{\prime} M_{i}+M_{i}\left(A_{i}+B_{i} K_{i}\right)+\mathcal{E}_{i}(M)<0, \quad i=1, \ldots, N
$$

c) For some $\mathcal{K}=\left(K_{1}, \ldots, K_{N}\right)$, we have that $E\left(\|x(t)\|^{2}\right) \leq a e^{b t}$ for some a $\geq 0, b<0$, where $x(t)$ is given by (2.3) with $u(t)=K_{\theta(t)} x(t)$.

Remark 2.1: From the above proposition, we have that if $\mathcal{K}=\left(K_{1}, \ldots, K_{N}\right)$ stabilizes $(\mathcal{A}, \mathcal{B})$ in the mean square sense, then for some $\mathcal{M}=\left(M_{1}, \ldots, M_{N}\right), M_{i}>0$ for $i=1, \ldots, N$, we have that

$$
\left(\widehat{A}_{i}+B_{i} K_{i}\right)^{\prime} M_{i}+M_{i}\left(\widehat{A}_{i}+B_{i} K_{i}\right)<0
$$

where $\widehat{A}_{i}:=A_{i}+\frac{1}{2} \lambda_{i i} \cdot I, i=1, \ldots, N$, and therefore, $\left(\widehat{A}_{i}, B_{i}\right)$ is stabilizable and $K_{i}$ stabilizes $\left(\widehat{A}_{i}, B_{i}\right)$, $i=1, \ldots, N$.

\subsection{Conditions for Mean Square Stabilizing Solution}

We shall now derive some new conditions for the existence of the mean square stabilizing solution of the ISARE. The next result is immediate from [11 theo 5], after noticing that $(\mathcal{A}, \mathcal{B})$ is mean square stabilizable if and only if $(\overline{\mathcal{A}}, \mathcal{B})$ is mean square stabilizable, and that $(2.1)$ can be rewritten in the equivalent form:

$$
\bar{A}_{i}^{\prime} X_{i}+X_{i} \bar{A}_{i}+\mathcal{E}_{i}(X)+\bar{C}_{i}^{\prime} \bar{C}_{i}-X_{i} B_{i}\left(D_{i}^{\prime} D_{i}\right)^{-1} B_{i}^{\prime} X_{i}=0
$$

Proposition 2.2: Suppose that $(\mathcal{A}, \mathcal{B})$ is mean square stabilizable and $\left(\bar{C}_{i}, \bar{A}_{i}\right)$ is observable for each $i=1, \ldots, N$. Then there exists a unique positive semi-definite solution $\mathcal{P}=\left(P_{1}, \ldots, P_{N}\right)$ to the ISARE (2.1). Moreover $\mathcal{P}$ is the positive definite mean square stabilizing solution of (2.1).

We shall now apply the results of [7] to derive new conditions for the existence of the mean square stabilizing solution of (2.1).

Theorem 2.1: Suppose that $(\mathcal{A}, \mathcal{B})$ is mean square stabilizable and $(\overline{\mathcal{C}}, \overline{\mathcal{A}})$ is mean square detectable. Then there exists a unique positive semi-definite solution $\mathcal{P}=\left(P, \ldots, P_{N}\right)$ to the ISARE (2.1). Moreover $\mathcal{P}$ is the mean square stabilizing solution of (2.1).

Proof: Define

$$
\begin{array}{r}
\mathbb{B}=\left\{\mathfrak{X}=\left(\begin{array}{ccc}
X_{1} & \cdots & 0 \\
\vdots & \ddots & \vdots \\
0 & \cdots & X_{N}
\end{array}\right), X_{i}\right. \text { is a square matrix } \\
\text { with the same dimension as } \left.A_{i}, i=1, \ldots, N\right\},
\end{array}
$$




$$
\text { and set } \begin{aligned}
\mathbb{B}^{+}=\{\mathfrak{X} & \left.=\left(\begin{array}{ccc}
X_{1} & \cdots & 0 \\
\vdots & \ddots & \vdots \\
0 & \cdots & X_{N}
\end{array}\right) \in \mathbb{B} \text { such that } X_{i} \geq 0, i=1, \ldots, N\right\} \text {. Define also } \\
\mathfrak{A} & =\left(\begin{array}{cccc}
\bar{A}_{1}+\frac{1}{2} \lambda_{11} \cdot I & \cdots & 0 \\
\vdots & \ddots & \vdots \\
& 0 & \cdots & \bar{A}_{N}+\frac{1}{2} \lambda_{N N} \cdot I
\end{array}\right), \mathfrak{G}=\left(\begin{array}{ccc}
B_{1} & \cdots & 0 \\
\vdots & \ddots & \vdots \\
0 & \cdots & B_{N}
\end{array}\right) \\
\mathfrak{C} & =\left(\begin{array}{ccc}
\bar{C}_{1} & \cdots & 0 \\
\vdots & \ddots & \vdots \\
0 & \cdots & \bar{C}_{N}
\end{array}\right), \mathfrak{D}=\left(\begin{array}{ccc}
D_{1} & \cdots & 0 \\
\vdots & \ddots & \vdots \\
0 & \cdots & D_{N}
\end{array}\right)
\end{aligned}
$$

and the linear operator $\Pi$ from $\mathbb{B}$ into $\mathbb{B}$ as,

$$
\Pi(\mathfrak{X})=\left(\begin{array}{ccc}
\sum_{\substack{j=1 \\
j \neq 1}}^{N} \lambda_{1 j} X_{j} & \cdots & 0 \\
\vdots & \ddots & \vdots \\
0 & \cdots & \sum_{\substack{j=1 \\
j \neq N}}^{N} \lambda_{N j} X_{j}
\end{array}\right) \text { for } \mathfrak{X} \in \mathbb{B} .
$$

It is easy to verify that $\Pi$ is actually a positive semi-definite operator, that is, $\Pi$ maps $\mathbb{B}^{+}$into $\mathbb{B}^{+}$. Moreover, equation (2.1) can be rewritten as the following linearly perturbed algebraic Riccati equation (LPARE),

$$
\mathfrak{A}^{\prime} \mathfrak{X}+\mathfrak{X A}+\Pi(\mathfrak{X})+\mathfrak{C}^{\prime} \mathfrak{C}-\mathfrak{X} \mathfrak{G}\left(\mathfrak{D}^{\prime} \mathfrak{D}\right)^{-1} \mathfrak{G}^{\prime} \mathfrak{X}=0
$$

where $\mathfrak{X} \in \mathbb{B}$. Also it is easy to check that the concepts of $(\mathcal{A}, \mathcal{B})$ mean square stabilizable and $(\overline{\mathcal{C}}, \overline{\mathcal{A}})$ mean square detectable are equivalent to the concepts of $(\mathfrak{A}, \mathfrak{G})$ MS-stabilizable and $(\mathfrak{C}, \mathfrak{A})$ MSdetectable respectively in the sense defined in [7]. Therefore the above framework fits the setup considered for general LPARE defined in [7]. We can thus use Theorem 4.1 of [7] to get the desired resulE]

We can weaken the condition of observability of each pair $\left(\bar{C}_{i}, \bar{A}_{i}\right), i=1, \ldots, N$ required in Proposition 2.2 by requiring only observability of each mode with real part greater than $-\frac{1}{2} \lambda_{i i}$ (which are positive) of the pair $\left(\bar{C}_{i}, \bar{A}_{i}\right), i=1, \ldots, N$.

Theorem 2.2: Suppose that $(\mathcal{A}, \mathcal{B})$ is mean square stabilizable and for each $i=1, \ldots, N,\left(\bar{C}_{i}, \bar{A}_{i}\right)$ has no unobservable mode with real part greater than $-\frac{1}{2} \lambda_{i i}$. Then there exists the mean square stabilizing positive definite solution $\mathcal{P}=\left(P, \ldots, P_{N}\right)$ to the ISARE (2.1).

Proof: Note that the hypothesis that for each $i=1, \ldots, N,\left(\bar{C}_{i}, \bar{A}_{i}\right)$ has no unobservable mode with real part greater than $-\frac{1}{2} \lambda_{i i}$ is equivalent to $(\mathfrak{C}, \mathfrak{A})$ not having unobservable mode in the closed left half plane. Applying Theorem 4.4of [7] we get the desired result.

Therefore Theorem 2.2 weakens Proposition 2.2 for the existence of the mean square stabilizing solution of (2.1) in the sense that only observability of the modes of $\left(\bar{C}_{i}, \bar{A}_{i}\right)$ with real part greater than or equal to $-\frac{1}{2} \lambda_{i i}$ are required, for each $i=1, \ldots, N$. Note however that, unlike Proposition 2.2, uniqueness among the positive semi-definite solutions of (2.1) cannot be established in general under the conditions of Theorem 2.2. Since we will need this uniqueness throughout this paper, we shall be working 
with either one of the following hypothesis:

$$
\begin{aligned}
& \text { H1) }(\overline{\mathcal{C}}, \overline{\mathcal{A}}) \text { mean square detectable, or } \\
& \text { H2) for each } i=1, \ldots, N,\left(\bar{C}_{i}, \bar{A}_{i}\right) \text { is observable. }
\end{aligned}
$$

Clearly H1) does not imply $\mathrm{H} 2$ ) as can be immediately seen for the pure deterministic case. Conversely it can be shown that H2) does not imply H1) either, so that we cannot say in general that H1) (or H2)) is stronger or weaker than $\mathrm{H} 2$ ) (or $\mathrm{H} 1)$ ).

\subsection{Preliminary Results for the Uncoupled Algebraic Riccati Equations}

For solving the ISARE in (2.1) via uncoupled algebraic Riccati equation (ARE), we need to consider the following type of equations in $X$ :

$$
\widehat{A}^{\prime} X+X \widehat{A}+\left(C^{\prime} C+Y\right)-(X B+C D)\left(D^{\prime} D\right)^{-1}\left(B^{\prime} X+D^{\prime} C\right)=0
$$

where $\widehat{A}=A+\nu I, \nu \leq 0, Y \geq 0$ and $D^{\prime} D>0$. For solving the ISARE in (2.1) via uncoupled Lyapunov equation, the following type of equations in $X$ is considered:

$$
(\widehat{A}+B K Y X+X(\widehat{A}+B K)+Y+(C+D K Y(C+D K)=0
$$

where $\widehat{A}=A+\nu I, \nu \leq 0, \quad Y \geq 0, \quad$ and $D^{\prime} D>0$. Later we set in (2.5) and (2.6): $\nu=\lambda_{i i} / 2$, $\widehat{A}=\widehat{A}_{i}=A_{i}+\frac{\lambda_{i i}}{2} I, B=B_{i}, C=C_{i}, D=D_{i}$ for each.

Remark 2.2: Note that $(\mathcal{A}, \mathcal{B})$ mean square stabilizable implies, from Proposition 2.1 and Remark 2.1, that $\left(\widehat{A}_{i}, B_{i}\right)$ is stabilizable and if condition $\left.\mathrm{H} 1\right)$ holds then, by similar arguments, we have that $\left(\left(I-D_{l}\left(D_{i}^{\prime} D_{i}\right)^{-1} D_{i}^{\prime}\right) C_{i}, \widehat{A}_{i}-B_{i}\left(D_{i}^{\prime} D_{i}\right)^{-1} D_{i}^{\prime} C_{i}\right)$ is detectable for each $i=1, \ldots, N$. For H2), if $\left(\bar{C}_{i}, \bar{A}_{i}\right)$ is observable for each $i=1, \ldots, N$ then it is clear that $\left(\bar{C}_{i}, \bar{A}_{i}+\frac{\lambda_{i i}}{2} I\right)=\left(\left(I-D_{i}\left(D_{i} D_{i}\right)^{-1} D_{i}^{\prime}\right) C_{i}\right.$, $\left.\widehat{A}_{i}-B_{i}\left(D_{i}^{\prime} D_{i}\right)^{-1} D_{i}^{\prime} C_{i}\right)$ is also observable. In conclusion, we have that if $(\mathcal{A}, \mathcal{B})$ mean square stabilizable and condition H1) or H2) in section 2.2 is satisfied then for each $i=1, \ldots, N,\left(\widehat{A}_{i}, B_{i}\right)$ is stabilizable and $\left(\left(I-D_{i}\left(D_{i}^{\prime} D_{i}\right)^{-1} D_{i}^{\prime}\right) C_{i}, \widehat{A}_{i}-B_{i}\left(D_{i}^{\prime} D_{i}\right)^{-1} D_{i}^{\prime} C_{i}\right)$ is detectable. Finally, we mention that if $\left(\widehat{A}_{i}, B_{i}\right)$ is stabilizable, $\left(\widehat{A}_{i}-B_{i}\left(D_{i}^{\prime} D_{i}\right)^{-1} D_{i}^{\prime} C_{i}, B_{i}\right)$ also is.

We need the results in the sequel, see the Appendix for the proof.

\section{Proposition 2.3:}

(i) Suppose that $(\widehat{A}, B)$ is stabilizable and $\left(\left(I-D\left(D^{\prime} D\right)^{-1} D^{\prime}\right) C, \widehat{A}-B\left(D^{\prime} D\right)^{-1} D^{\prime} C\right)$ is detectable. Then there exists a unique positive semi-definite solution to (2.5) and this solution is the stabilizing solution. Let $\bar{X}$ and $\tilde{X}$ be the correspondent positive definite solutions of (2.5), when $Y=\bar{Y}$ and $Y=\tilde{Y}$, respectively. If $\bar{Y} \leq \tilde{Y}$, then $\bar{X} \leq \tilde{X}$.

(ii) If $(\widehat{A}+B K)$ is stable, there exists a unique positive semi-definite solution to (2.6). Suppose that $(\widehat{A}+B K)$ is stable and let $\tilde{X} \geq 0$ be the solution of (2.6) when $Y=\tilde{Y}$. If $\left(\left(I-D\left(D^{\prime} D\right)^{-1} D^{\prime}\right) C\right.$, $\left.\widehat{A}-B\left(D^{\prime} D\right)^{-1} D^{\prime} C\right)$ is detectable, then $\widehat{A}+B \tilde{K}$ is stable, where $\tilde{K}=-\left(D^{\prime} D\right)^{-1}\left(B^{\prime} \tilde{X}+D C\right)$. Moreover, the solution $\bar{X}$ of (2.6) when $K=\tilde{K}$ and $Y=\bar{Y} \leq \tilde{Y}$, is such that $\bar{X} \leq \tilde{X}$.

\section{The Main Result}

Our purpose is to attain the solution of (2.1) by iterations on a set of decoupled Riccati equations. We will present two algorithms which will be related to truncated quadratic optimal control problems, each one 
associated with a different sequence of stopping times as terminal time. We shall call these methods Method I and Method II, the later based on existing results, see [1]. We start with Method I:

R1. Set $\mathcal{X}^{0}=\left(X_{1}^{0}, \ldots, X_{N}^{0}\right)$ arbitrary, with $X_{i}^{0} \geq 0, i=1, \ldots, N$;

R2. For $k=1,2, \ldots$ and $i=1, \ldots, N$, calculate recursively,

$$
\widehat{A}_{i}^{\prime} X_{i}^{k}+X_{i}^{k} \widehat{A}_{i}+C_{i}^{\prime} C_{i}+\mathcal{E}_{i}\left(\tilde{X}_{i}^{k}\right)-\left(X_{i}^{k} B_{i}+C_{i}^{\prime} D_{i}\right)\left(D_{i}^{\prime} D_{i}\right)^{-1}\left(B_{i}^{\prime} X_{i}^{k}+D_{i}^{\prime} C_{i}\right)=0
$$

where $\tilde{X}_{i}^{k}=\left(\tilde{X}_{i 1}^{k}, \ldots, \tilde{X}_{i N}^{k}\right)$ is defined as

$$
\tilde{X}_{i j}^{k}= \begin{cases}X_{j}^{k}, & \text { for } j=1, \ldots, i-1 \\ 0, & \text { for } j=i \\ X_{j}^{k-1}, & \text { for } j=i+1, \ldots, N\end{cases}
$$

A variant of Method I regarding the form (3.2) of carrying new updates, would be the following. For each $i$ set in the algorithm:

$$
\tilde{X}_{i j}^{k}= \begin{cases}0, & \text { for } j=i \\ X_{j}^{k-1}, & \text { otherwise. }\end{cases}
$$

For future reference, we shall call this Method II. We also denote by

$$
\mathcal{S}^{k}\left(\mathcal{X}^{0}\right)=\left(S_{1}^{k}\left(\mathcal{X}^{0}\right), \ldots, S_{N}^{k}\left(\mathcal{X}^{0}\right)\right) \text { and } \mathcal{R}^{k}\left(\mathcal{X}^{0}\right)=\left(R_{1}^{k}\left(\mathcal{X}^{0}\right), \ldots, R_{N}^{k}\left(\mathcal{X}^{0}\right)\right),
$$

the sequences generated by Methods I and II when $S^{0}\left(\mathcal{X}^{0}\right)=\mathcal{X}^{0}$ and $\mathcal{R}^{0}\left(\mathcal{X}^{0}\right)=\mathcal{X}^{0}$ respectively

There are other classes of methods for solving (2.1) in the literature that we wish to consider. A class of methods based on recursions on a set of decoupled Lyapunov equations, cf. [9], [10] is as follows.

L1.[initialization] Find $\mathcal{F}^{0}=\left(F_{1}^{0}, \ldots, F_{N}^{0}\right)$ such that

$$
\widehat{A}_{i}+B_{i} F_{i}^{0}
$$

is stable for each $i=1, \ldots, N$. Set $\mathcal{X}^{0}=\left(X_{1}^{0} \ldots, X_{N}^{0}\right)$ arbitrary, with $X_{i}^{0} \geq 0, i=1, \ldots, N$.

L2. For $k=1,2, \ldots$, calculate recursively,

$$
\begin{aligned}
& {\left[\widehat{A}_{i}+B_{i} F_{i}^{k-1}\right]^{\prime} X_{i}^{k}+X_{i}^{k}\left[\widehat{A}_{i}+B_{i} F_{i}^{k-1}\right]+\mathcal{E}_{i}\left(\tilde{X}_{i}^{k}\right)+\left(C_{i}+D_{i} F_{i}^{k-1}\right)^{\prime}\left(C_{i}+D_{i} F_{i}^{k-1}\right)=0} \\
& \text { with } F_{i}^{k-1}=-\left(D^{\prime} D\right)^{-1}\left(B_{i}^{\prime} X_{i}^{k-1}+D_{i}^{\prime} C_{i}\right), k=2,3, \ldots
\end{aligned}
$$

where $\tilde{X}_{i}^{k}=\left(\tilde{X}_{i 1}^{k}, \ldots, \tilde{X}_{i N}^{k}\right)$ is defined by (3.2) or (3.3). Regarding these update forms, we name these variants Method III and IV, respectively. For a fixed $\mathcal{F}^{0}=\left(F_{1}^{0}, \ldots, F_{N}^{0}\right)$ satisfying L1, we shall denote by

$$
\mathcal{Q}^{k}\left(\mathcal{X}^{0}\right)=\left(Q_{1}^{k}\left(\mathcal{X}^{0}\right), \ldots, Q_{N}^{k}\left(\mathcal{X}^{0}\right)\right) \quad \text { and } \quad \mathcal{Z}^{k}\left(\mathcal{X}^{0}\right)=\left(Z_{1}^{k}\left(\mathcal{X}^{0}\right), \ldots, Z_{N}^{k}\left(\mathcal{X}^{0}\right)\right),
$$

the sequences generated by Methods III and IV when $\mathcal{Q}^{0}\left(\mathcal{X}^{0}\right)=\mathcal{X}^{0}$ and $\mathcal{Z}^{0}\left(\mathcal{X}^{0}\right)=\mathcal{X}^{0}$ respectively. 
Finally, a third class of methods for solving (2.1) we consider is based on LMI's as it appears in [2]. Here we name it Method V, and it applies to the present problem with the following formulation:

$$
\text { maximize trace. }\left\{X_{1}+\cdots+X_{N}\right\}
$$

subject to

$$
\left(\begin{array}{cc}
\widehat{A}_{i}^{\prime} X_{i}+X_{i} \widehat{A}_{i}+\mathcal{E}_{i}\left(\tilde{X}_{i}\right)+C_{i}^{\prime} C_{i} & X_{i} B_{i}+C_{i}^{\prime} D_{i} \\
B_{i}^{\prime} X_{i}+D_{i}^{\prime} C_{i} & D_{i}^{\prime} D_{i}
\end{array}\right) \geq 0, \quad X_{i}=X_{\imath}^{\prime} i=1, \ldots, N
$$

We shall make comparisons between the methods described from an analytical point of view (see Theorems 3.1 and 3.2), although comparisons between method $\mathrm{V}$ and the previous ones are restricted to numerical performance comparisons, see section 4.

The form of taking the updates into account in Methods I and III is new for continuous time MJLS; for the counterpart of Method I for discrete-time problems, see [6].

Remark 3.1: Method II was previously studied in [1], but the convergence is assured with some restrictions on the initial value $\mathcal{X}^{0}$, namely,

C1. $\mathcal{X}^{0}=0$, or

$$
\text { C2. } \mathcal{X}^{0} \geq \mathcal{P} \text { and } \mathcal{X}^{0} \geq \mathcal{X}^{1} \text {. }
$$

Conditions (C1) or (C2) are not necessary for convergence of Methods I and II, as shown here. Regarding Methods I and II, if (C1) or (C2) is satisfied, the sequence produced is monotone, see respectively, Lemma 3.1 or Lemma 3.2.

Remark 3.2: The class of Lyapunov iteration methods is derived from the method of Kleinman for ARE's [12] and the set of interconnected Lyapunov equations introduced by Wonham [15],[16]. An initial controller that stabilizes all modes $\left(\widehat{A}_{i}, B_{i}\right)$ is mandatory, as in the step (L1) above. Method IV appears in [9] (assuming that $\left.C_{i}^{\prime} D_{i}=0, \forall i\right)$, and the results relies on a hard to check condition introduced by Wonham, to make sure that there exists the mean square stabilizing positive semi-definite solution to (2.1). Here this condition is replaced by mean square stabilizability of $(\mathcal{A}, \mathcal{B})$ and condition $\mathrm{H} 1)$ or $\mathrm{H} 2)$ in section 2.2. Condition (C2) is required in Method IV to guarantee that the sequence produced is monotone, see [9] or [10].

The main results read as follows.

Theorem 3.1: Suppose that $(\mathcal{A}, \mathcal{B})$ is mean square stabilizable and condition $\mathrm{H} 1)$ or $\mathrm{H} 2)$ in section 2.2 is satisfied. Then the sequences $\left\{X_{i}^{k}\right\}$ defined by Methods I and II are such that $X_{i}^{k} \rightarrow P_{i}$ as $k \rightarrow \infty$, for $i=1, \ldots, N$, where $\mathcal{P}=\left(P, \ldots, P_{N}\right)$ is the unique positive semi-definite solution of (2.1).

Theorem 3.2 [rate of convergence]: Suppose that the methods are initialized with the same value $\mathcal{X}^{0}$ :

(i) Method I has a larger rate of convergence than that of Method II;

(ii) Suppose that for some $\mathcal{F}^{0}=\left(F_{1}^{0}, \ldots, F_{N}^{0}\right)$ satisfying (L1) we have $\mathcal{X}^{0} \geq \mathcal{Z}^{1}\left(\mathcal{X}^{0}\right)$. Then

$$
\begin{aligned}
& \text { (a) } \mathcal{Q}^{k}\left(\mathcal{X}^{0}\right) \geq \mathcal{Q}^{k+1}\left(\mathcal{X}^{0}\right) \geq \mathcal{S}^{k+1}\left(\mathcal{X}^{0}\right) \geq 0 \\
& \text { (b) } \mathcal{Z}^{k}\left(\mathcal{X}^{0}\right) \geq \mathcal{Z}^{k+1}\left(\mathcal{X}^{0}\right) \geq \mathcal{R}^{k+1}\left(\mathcal{X}^{0}\right) \geq \mathcal{S}^{k+1}\left(\mathcal{X}^{0}\right) \geq 0 \\
& \text { (c) } \mathcal{R}^{k}\left(\mathcal{X}^{0}\right) \geq \mathcal{R}^{k+1}\left(\mathcal{X}^{0}\right) \geq 0, \mathcal{S}^{k}\left(\mathcal{X}^{0}\right) \geq \mathcal{S}^{k+1}\left(\mathcal{X}^{0}\right) \geq 0
\end{aligned}
$$

for all $k=0,1, \ldots$, with $\mathcal{Q}^{k}\left(\mathcal{X}^{0}\right) \downarrow \mathcal{P}, Z^{k}\left(\mathcal{X}^{0}\right) \downarrow \mathcal{P}, \mathcal{R}^{k}\left(\mathcal{X}^{0}\right) \downarrow \mathcal{P}$ and $\mathcal{S}^{k}\left(\mathcal{X}^{0}\right) \downarrow \mathcal{P}$ as $k \rightarrow \infty$. 
Theorem 3.3: Suppose that condition H1) or H2) in section 2.2 is satisfied. Then the sequences $\left\{X_{i}^{k}\right\}$ defined by Method I or II converges if and only if $(\mathcal{A}, \mathcal{B})$ is mean square stabilizable.

The proof of the Theorems relies on the next Lemmas.

Lemma 3.1: Suppose that $(\mathcal{A}, \mathcal{B})$ is mean square stabilizable and condition $\mathrm{H} 1)$ or $\mathrm{H} 2)$ in section 2.2 is satisfied. With $\mathcal{X}^{0}=0$, we have for $k=0,1,2, \ldots$. , that

$$
\begin{aligned}
& \text { (i) } \mathcal{R}^{k}\left(\mathcal{X}^{0}\right) \leq \mathcal{S}^{k}\left(\mathcal{X}^{0}\right) \\
& \text { (ii) } \mathcal{S}^{k}\left(\mathcal{X}^{0}\right) \leq \mathcal{S}^{k+1}\left(\mathcal{X}^{0}\right) \leq \mathcal{P} \\
& \text { (iii) } \mathcal{R}^{k}\left(\mathcal{X}^{0}\right) \leq \mathcal{R}^{k+1}\left(\mathcal{X}^{0}\right) \leq \mathcal{P}
\end{aligned}
$$

and $\mathcal{S}^{k}\left(\mathcal{X}^{0}\right) \uparrow P_{i}, \mathcal{R}^{k}\left(\mathcal{X}^{0}\right) \uparrow P_{i}$ as $k \rightarrow \infty$, for $i=1, \ldots, N$, where $\mathcal{P}=\left(P_{1}, \ldots, P_{N}\right)$ is the unique positive semi-definite solution of (2.1).

Proof: First of all, notice that from the hypothesis and the results in section 2, there exists a unique positive semi-definite solution $\mathcal{P}=\left(P_{1}, \ldots, P_{N}\right)$ to the ISARE $(2.1)$, and thus satisfies (recall that $\left.\widehat{A}_{i}=A_{i}+\frac{\lambda_{i i}}{2} I\right)$

$$
\widehat{A}_{i}^{\prime} P_{i}+P_{i} \widehat{A}_{i}+C_{i} C_{i}+\sum_{j=1, j \neq i}^{N} \lambda_{i j} P_{j}-\left(P_{i} B_{i}+C_{i} D_{i}\right)\left(D_{i}^{\prime} D_{i}\right)^{-1}\left(B_{i}^{\prime} P_{i}+D_{i}^{\prime} C_{i}\right)=0
$$

Moreover, as mentioned in section 2.3, we have that $\left(\widehat{A}_{i}, B_{i}\right)$ is stabilizable and ( $\left.\left(I-D_{l}\left(D_{i} D_{i}\right)^{-1} D_{i}^{\prime}\right) C_{i}, \widehat{A}_{i}-B_{i}\left(D_{i}^{\prime} D_{i}\right)^{-1} D_{i}^{\prime} C_{i}\right)$ is detectable, $i=1, \ldots, N$ so that from Proposition 2.3 (i) and Remark 2.2 the Riccati equation (3.1) has a unique positive semi-definite solution and this solution is the stabilizing one. Set

$$
\mathcal{S}^{k}\left(\mathcal{X}^{0}\right)=\left(S_{1}^{k}, \ldots, S_{N}^{k}\right), \mathcal{R}^{k}\left(\mathcal{X}^{0}\right)=\left(R_{1}^{k}, \ldots, R_{N}^{k}\right) .
$$

Let us show by induction that for all $\ell=0,1,2, \ldots$

$$
0 \leq S_{i}^{k} \leq S_{i}^{k+1} \leq P_{i}, \text { where } k=\operatorname{int}\left(\frac{\ell}{N}\right), i=\ell-(k \times N)+1
$$

where $\operatorname{int}\left(\frac{\ell}{N}\right)$ is the integer part of the division of $\ell$ by $N$. At any iteration we have that

$$
\begin{aligned}
& \widehat{A}_{i}^{\prime} S_{i}^{r+1}+S_{i}^{r+1} \widehat{A}_{i}+C_{i} C_{i}-\left(S_{i}^{r+1} B_{i}+C_{i} D_{i}\right)\left(D_{i}^{\prime} D_{i}\right)^{-1}\left(B_{i}^{\prime} S_{i}^{r+1}+D_{i}^{\prime} C_{i}\right) \\
& +\sum_{j=1}^{i-1} \lambda_{i j} S_{j}^{r+1}+\sum_{j=i+1}^{N} \lambda_{i j} S_{j}^{r}=0
\end{aligned}
$$

and

$$
\begin{aligned}
& \widehat{A}_{i}^{\prime} S_{i}^{r}+S_{i}^{r} \widehat{A}_{i}+C_{i}^{\prime} C_{i}-\left(S_{i}^{r} B_{i}+C_{i}^{\prime} D_{i}\right)\left(D_{i}^{\prime} D_{i}\right)^{-1}\left(B_{i}^{\prime} S_{i}^{r}+D_{i}^{\prime} C_{i}\right) \\
& +\sum_{j=1}^{i-1} \lambda_{i j} S_{j}^{r}+\sum_{j=i+1}^{N} \lambda_{i j} S_{j}^{r-1}=0 .
\end{aligned}
$$

For $\ell=0$ (that is, $k=0, i=1$ ), (3.6) clearly holds from Proposition 2.3 (i) and equations (3.5) and (3.7), 
since $\sum_{j=2}^{N} \lambda_{1 j} P_{j} \geq 0$ and $S_{j}^{0}=0, P_{j} \geq 0 j=1, \ldots, N$. Suppose that for all $\ell=1, \ldots, \nu$, we have that (3.6) holds, and write $k=\operatorname{int}\left(\frac{\nu}{N}\right), \iota=\nu-(k \times N)+1$. Suppose first that $\iota<N$, so that $\operatorname{int}\left(\frac{\nu+1}{N}\right)=k$. Then from the induction hypothesis,

$$
\sum_{j=1}^{\iota} \lambda_{\iota+1 j} S_{j}^{k}+\sum_{j=\iota+2}^{N} \lambda_{\iota+1 j} S_{j}^{k-1} \leq \sum_{j=1}^{\iota} \lambda_{\iota+1 j} S_{j}^{k+1}+\sum_{j=\iota+2}^{N} \lambda_{\iota+1 j} S_{j}^{k} \leq \sum_{j=1, j \neq \iota}^{N} \lambda_{\iota+1 j} P_{j}
$$

and from Proposition 2.3 (i), equations (3.5), (3.7) and (3.8) with $i=\iota+1, r=k$, we have that

$$
S_{\iota+1}^{k} \leq S_{\iota+1}^{k+1} \leq P_{\iota+1}
$$

showing that (3.6) holds for $\nu+1=\iota+(k \times N)$. If $\iota=N$ then $\nu+1=(k+1) \times N$, and from the induction hypothesis,

$$
\sum_{j=2}^{N} \lambda_{1 j} S_{j}^{k} \leq \sum_{j=2}^{N} \lambda_{1 j} S_{j}^{k+1} \leq \sum_{j=2}^{N} \lambda_{1 j} P_{j}
$$

and from Proposition 2.3 (i), equations (3.5), (3.7) and (3.8) with $i=1, r=k+1$, we have that

$$
S_{1}^{k+1} \leq S_{1}^{k+2} \leq P_{\iota+1}
$$

showing that (3.6) holds for $\nu+1=(k+1) \times N$. By the same arguments we have that (iii) holds. Let us show (i) by induction, that is, for all $\ell=0,1,2, \ldots$, we have that

$$
0 \leq R_{i}^{k} \leq S_{i}^{k}, \text { where } k=\operatorname{int}\left(\frac{\ell}{N}\right), i=l-(k \times N)+1 .
$$

For $\ell=0$ the result clearly hods since $0=R_{1}^{0}=S_{1}^{0}$. Suppose that for all $\ell=1, \ldots, \nu$, we have that the result holds, and write $k=\operatorname{int}\left(\frac{\nu}{N}\right), i=\nu-(k \times N)+1$, and suppose that $\iota<N$. Then from (3.6) and the induction hypothesis,

$$
\sum_{j=1}^{\iota} \lambda_{\iota+1 j} R_{j}^{k}+\sum_{j=\iota+2}^{N} \lambda_{\iota}+1 j R_{j}^{k} \leq \sum_{j=1}^{\iota} \lambda_{\iota}+1 j S_{j}^{k}+\sum_{j=\iota+2}^{N} \lambda_{\iota+1 j} S_{j}^{k} \leq \sum_{j=1}^{\iota} \lambda_{\iota+1 j} S_{j}^{k+1}+\sum_{j=\iota+2}^{N} \lambda_{\iota+1 j} S_{j}^{k}
$$

and since

$$
\begin{aligned}
& \widehat{A}_{i}^{\prime} R_{i}^{r}+R_{i}^{r} \widehat{A}_{i}+C_{i}^{\prime} C_{i}-\left(R_{i}^{k} B_{i}+C_{i}^{\prime} D_{i}\right)\left(D_{i}^{\prime} D_{i}\right)^{-1}\left(B_{i}^{\prime} R_{i}^{k}+D_{i}^{\prime} C_{i}\right) \\
& +\sum_{j=1}^{i-1} \lambda_{i j} R_{j}^{r-1}+\sum_{j=i+1}^{N} \lambda_{i j} R_{j}^{r-1}=0
\end{aligned}
$$

we have from Proposition 2.3 (i), equation (3.8) and the equation above with $i=i+1, r=k$, that $R_{\iota+1}^{k} \leq S_{\iota+1}^{k}$, showing the result for $\nu+1=\iota+(k \times N)$. The case $\iota=N$ parallels the previous one, and we can conclude in a similar way that $R_{1}^{k+1} \leq S_{1}^{k+1}$ for $\nu+1=(k+1) \times N$.

Lemma 3.2: Suppose that $(\mathcal{A}, \mathcal{B})$ is mean square stabilizable and condition $\mathrm{H} 1)$ or $\mathrm{H} 2)$ in section 2.2 is satisfied. Consider $\mathcal{F}^{0}=\left(F_{1}^{0}, \ldots, F_{N}^{0}\right)$ satisfying (L1) and $\mathcal{X}^{0}$ such that $\mathcal{X}^{0} \geq \mathcal{Z}^{1}\left(\mathcal{X}^{0}\right)$. Then 


$$
\begin{aligned}
& \text { (a) } \mathcal{Q}^{k}\left(\mathcal{X}^{0}\right) \geq \mathcal{Q}^{k+1}\left(\mathcal{X}^{0}\right) \geq \mathcal{S}^{k+1}\left(\mathcal{X}^{0}\right) \geq 0 \\
& \text { (b) } \mathcal{Z}^{k}\left(\mathcal{X}^{0}\right) \geq \mathcal{Z}^{k+1}\left(\mathcal{X}^{0}\right) \geq \mathcal{R}^{k+1}\left(\mathcal{X}^{0}\right) \geq \mathcal{S}^{k+1}\left(\mathcal{X}^{0}\right) \geq 0 \\
& \text { (c) } \mathcal{R}^{k}\left(\mathcal{X}^{0}\right) \geq \mathcal{R}^{k+1}\left(\mathcal{X}^{0}\right) \geq 0, \mathcal{S}^{k}\left(\mathcal{X}^{0}\right) \geq \mathcal{S}^{k+1}\left(\mathcal{X}^{0}\right) \geq 0
\end{aligned}
$$

for all $k=0,1, \ldots$, with $\mathcal{Q}^{k}\left(\mathcal{X}^{0}\right) \downarrow \mathcal{P}, Z^{k}\left(\mathcal{X}^{0}\right) \downarrow \mathcal{P}, \quad \mathcal{R}^{k}\left(\mathcal{X}^{0}\right) \downarrow \mathcal{P}$ and $\mathcal{S}^{k}\left(\mathcal{X}^{0}\right) \downarrow \mathcal{P}$ as $k \rightarrow \infty, \mathcal{P}$ the unique positive semi-definite solution of (2.1).

Proof: First of all notice from Proposition 2.3 (ii) and Remark 2.2 that for each $k=0,1,2, \ldots, \widehat{A}_{i}+B_{i} F_{i}^{k}$ is stable, and thus the sequence in (L2) is well defined. Set

$$
\begin{aligned}
& \mathcal{Q}^{k}\left(\mathcal{X}^{0}\right)=\left(Q_{1}^{k}, \ldots, Q_{N}^{k}\right), \mathcal{Z}^{k}\left(\mathcal{X}^{0}\right)=\left(Z_{1}^{k}, \ldots, Z_{N}^{k}\right) \\
& \mathcal{S}^{k}\left(\mathcal{X}^{0}\right)=\left(S_{1}^{k}, \ldots, S_{N}^{k}\right), \mathcal{R}^{k}\left(\mathcal{X}^{0}\right)=\left(R_{1}^{k}, \ldots, R_{N}^{k}\right) .
\end{aligned}
$$

At any iteration we have that

$$
\begin{aligned}
& {\left[\widehat{A}_{i}+B_{i} F_{i}^{r-1}\right]^{\prime} Q_{i}^{r}+Q_{i}^{r}\left[\widehat{A}_{i}+B_{i} F_{i}^{r-1}\right]+\left(C_{i}+D_{i} F_{i}^{r-1}\right)^{\prime}\left(C_{i}+D_{i} F_{i}^{r-1}\right)} \\
& +\sum_{j=1}^{i-1} \lambda_{i j} Q_{j}^{r-1}+\sum_{j=i+1}^{N} \lambda_{i j} Q_{j}^{r-1}= \\
& \widehat{A}_{i}^{\prime} Q_{i}^{r}+Q_{i}^{r} \widehat{A}_{i}+C_{i}^{\prime} C_{i}-\left(Q_{i}^{r} B_{i}+C_{i}^{\prime} D_{i}\right)\left(D_{i}^{\prime} D_{i}\right)^{-1}\left(B_{i}^{\prime} Q_{i}^{r}+D_{i}^{\prime} C_{i}\right) \\
& +\sum_{j=1}^{i-1} \lambda_{i j} Q_{j}^{r-1}+\sum_{j=i+1}^{N} \lambda_{i j} Q_{j}^{r-1}+\left(F_{i}^{r}-F_{i}^{r-1}\right)^{\prime}\left(D_{i}^{\prime} D_{j}\left(F_{i}^{r}-F_{i}^{r-1}\right)=0\right.
\end{aligned}
$$

where

$$
F_{i}^{\ell}=-\left(D^{\prime} D\right)^{-1}\left(B_{i}^{\prime} Q_{i}^{\ell}+D_{i}^{\prime} C_{i}\right), \ell \geq 1
$$

Similarly,

$$
\begin{aligned}
& {\left[\widehat{A}_{i}+B_{i} G_{i}^{r-1}\right]^{\prime} Z_{i}^{r}+Z_{i}^{r}\left[\widehat{A}_{i}+B_{i} G_{i}^{r-1}\right]+\left(C_{i}+D_{i} G_{i}^{r-1}\right)^{\prime}\left(C_{i}+D_{i} G_{i}^{r-1}\right)} \\
& +\sum_{j=1}^{i-1} \lambda_{i j} Z_{j}^{r-1}+\sum_{j=i+1}^{N} \lambda_{i j} Z_{j}^{r-1}= \\
& \widehat{A}_{i}^{\prime} Z_{i}^{r}+Z_{i}^{r} \widehat{A}_{i}+C_{i}^{\prime} C_{i}-\left(Z_{i}^{r} B_{i}+C_{i} D_{i}\right)\left(D_{i}^{\prime} D_{i}\right)^{-1}\left(B_{i}^{\prime} Z_{i}^{r}+D_{i}^{\prime} C_{i}\right) \\
& +\sum_{j=1}^{i-1} \lambda_{i j} Z_{j}^{r-1}+\sum_{j=i+1}^{N} \lambda_{i j} Z_{j}^{r-1}+\left(G_{i}^{r}-G_{i}^{r-1}\right)^{\prime}\left(D_{i}^{\prime} D_{j}\right)\left(G_{i}^{r}-G_{i}^{r-1}\right)=0
\end{aligned}
$$

where

$$
G_{i}^{\ell}=-\left(D^{\prime} D\right)^{-1}\left(B_{i}^{\prime} Z_{i}^{\ell}+D_{i}^{\prime} C_{i}\right), \ell \geq 1, G_{i}^{0}=F_{i}^{0} .
$$

From the hypothesis made, we have that $Z_{i}^{1} \leq Z_{i}^{0}=X_{i}^{0}=Q_{i}^{0}$ and $Q_{1}^{1}=Z_{1}^{1}$, since $G_{1}^{0}=F_{1}^{0}$. Moreover we have from Proposition 2.3 (ii) and equations (3.11), (3.12) that $Q_{2}^{1} \leq Z_{2}^{1} \leq Z_{2}^{0}=Q_{2}^{0}$ (since $G_{2}^{0}=F_{2}^{0}$ ), and similarly, $Q_{i}^{1} \leq Z_{i}^{1} \leq Z_{i}^{0}=Q_{i}^{0}$ (since $G_{i}^{0}=F_{i}^{0}$ ), for $i=1, \ldots, N$. From equation (3.11), Proposition 2.3 (ii), and induction arguments similar to those presented in the proof of Lemma 3.1 we can show that $Q_{i}^{k+1} \leq Q_{i}^{k}$. From equations (3.8), (3.11), Proposition 2.3 (i), and induction arguments similar to those in the proof of Lemma 3.1 we get that $S_{i}^{k} \leq Q_{i}^{k}$ (recall that $S_{i}^{0}=X_{i}^{0}=Q_{i}^{0}$ ). In a similar way, using equations 
(3.8), (3.9) and (3.12), Proposition 2.3, and the arguments of the proof of Lemma 3.1 we can show that

$$
Z_{i}^{k} \geq Z_{i}^{k+1} \geq R_{i}^{k+1} \geq S_{i}^{k+1}, R_{i}^{k} \geq R_{i}^{k+1}, S_{i}^{k} \geq S_{i}^{k+1}
$$

completing the proof of (3.10).Taking the limit as $k \rightarrow \infty$, we get that $Q_{i}^{k} \downarrow Q_{i}$ for some $Q_{i} \geq 0$, $i=1, \ldots, N$. But taking the limit in equation (3.11) we get that $\mathcal{Q}=\left(Q_{1}, \ldots, Q_{N}\right)$ will be a positive semidefinite solution of (2.1), and from the uniqueness established in Section 2 it follows that $\mathcal{Q}=\mathcal{P}$. Similar arguments hold for the sequences $Z_{i}^{k}, R_{i}^{k}, S_{i}^{k}$.

Remark 3.3: It is immediate to check from Lemma 2.1 or 2.2 that the results also holds if, instead of solving the ARE's for $i$ going from 1 to $N$, we follow an arbitrary sequence $\left\{i_{1}, \ldots, i_{N}\right\}$ (it is just a matter of relabel the states). In fact we could consider any infinite sequence of states $I=\left\{i_{\eta}, \eta=1,2, \ldots ; i_{\eta}=i\right.$ infinitely often for each $i \in\{, \ldots, N\}\}$.

In Lemma 3.1 we require the condition $(\mathrm{C} 1)$, namely, $\mathcal{X}^{0}=0$, and in Lemma 3.2 the condition $(\mathrm{C} 2)$ is employed. However, we can generalize the result regarding the convergence of Methods I and II for any initial condition $\mathcal{X}^{0}$, and as a byproduct, Lemma 3.3 also provides a form to compare the rate of convergence of methods with updates (3.2) and (3.3).

We can show that Methods I and II are based on approximations via dynamic programming, involving a.s. finite horizon quadratic control problems, with some increasing sequence of stopping times as terminal time. Let us show this for Method II; for the others the arguments are similar. Define the following sequence of $\left\{\Im_{\mathrm{t}}\right\}$-stopping times $\{\tau(k): k=0,1, \ldots\}$ :

$$
\tau(0):=0, \quad \tau(k):=\min \left\{t>\tau(k-1): \theta(t) \neq \theta\left(t^{-}\right)\right\}
$$

$\left(\theta\left(t^{-}\right):=\lim _{s \uparrow t} \theta(s)\right)$, and set,

$$
J^{k}\left(x_{0}, \theta_{0}\right):=\min E\left\{\int_{0}^{\tau(k)}\left\|C_{\theta(t)} x(t)+D_{\theta(t)} u(t)\right\|^{2} d t+\left\|\left(X_{\theta(\tau(k))}^{0}\right)^{1 / 2} x(\tau(k))\right\|^{2}\right\}
$$

where the minimization is over $\{u(t) ; 0 \leq t \leq \tau(k)\}$.

Lemma 3.3: For each $k=0,1, \ldots$, and system (2.3) with a real vector $x_{0}$ and $\theta_{0}=i$,

$$
x_{0}^{\prime} R_{i}^{k}\left(\mathcal{X}^{0}\right) x_{0}=J^{k}\left(x_{0}, i\right)
$$

Proof: Let us apply induction on $k$. For $k=0$, the result is true by definition. Suppose it holds for $k$. Since $\{\theta(t), x(t): t \geq 0\}$ is a strong Markov process, we apply standard results on dynamic programming to obtain the following Bellman equation:

$$
J^{k+1}\left(x_{0}, \theta_{0}\right)=\min E_{x_{0}, \theta_{0}}\left\{\int_{0}^{\tau(1)}\left\|C_{\theta(t)} x(t)+D_{\theta(t)} u(t)\right\|^{2} d t+\left\|\left(R_{\theta(\tau(1))}^{k}\left(\mathcal{X}^{0}\right)\right)^{1 / 2} x(\tau(1))\right\|^{2}\right\} .
$$

Let $s \wedge t$ indicates $\min \{s, t\}$, and denote by $t \rightarrow x_{i}(t)$ the trajectories defined by $\dot{x}_{i}(t)=A_{i} x(t)+$ $B_{i} u(t)$ with $x_{i}(0)=x_{0} \in \mathbb{R}^{n}$, for each $i=1, \ldots, N$. We can write 


$$
\begin{aligned}
& E_{x_{0}, \theta_{0}}\left\{\int_{0}^{\tau(1) \wedge t}\left\|C_{\theta(s)} x(s)+D_{\theta(s)} u(s)\right\|^{2} d s\right\}=
\end{aligned}
$$

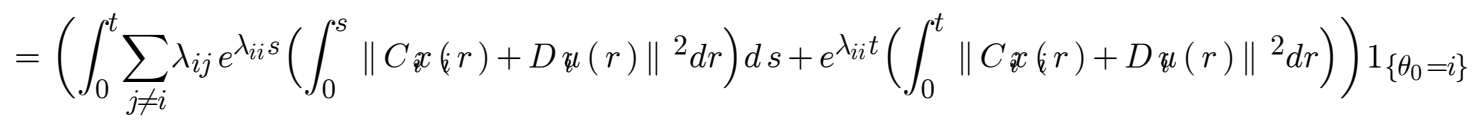

$$
\begin{aligned}
& =\left(\int_{0}^{t}-\lambda_{i i} e^{\lambda_{i i} s}\left(\int_{0}^{s}\left\|C_{x}(r)+D u(r)\right\|^{2} d r\right) d s+e^{\lambda_{i i} t}\left(\int_{0}^{t}\left\|C_{x}(\xi r)+D u(r)\right\|^{2} d r\right)\right) 1_{\left\{\theta_{0}=i\right\}} \\
& \left.=\int_{0}^{t}\left(e^{\lambda_{i i} r}-e^{\lambda_{i i} t}\right)\left\|C x\left({ }_{i} r\right)+D \underline{u}(r)\right\|^{2} d r+e^{\lambda_{i i} t}\left(\int_{0}^{t}\left\|C x(\xi r)+D_{i} u(r)\right\|^{2} d r\right)\right) 1_{\left\{\theta_{0}=i\right\}} \\
& =\int_{0}^{t} e^{\lambda_{i i} s}\|C x(s)+D u(s)\|^{2} d s \cdot 1_{\left\{\theta_{0}=i\right\}} \text {. }
\end{aligned}
$$

Define $V_{i}=R_{i}^{k+1}\left(\mathcal{X}^{0}\right)$ and $V_{j}=R_{j}^{k}\left(\mathcal{X}^{0}\right)$ for $j \neq i$. We have that

$$
\begin{gathered}
E_{x_{0}, \theta_{0}}\left\{x(\tau(1) \wedge t)^{\gamma} V_{\theta(\tau(1) \wedge t)} x(\tau(1) \wedge t)-x_{0}^{\prime} V_{\theta_{0}} x_{0}\right\} \\
=E_{x_{0}, \theta_{0}}\left\{x(\tau(1))^{\gamma} V_{\theta(\tau(1))} x(\tau(1)) 1_{\{\tau(1) \leq t\}}+x(t)^{\gamma} V_{\theta(t)} x(t) 1_{\{\tau(1)>t\}}-x_{0}^{\prime} V_{\theta_{0}} x_{0}\right\} \\
=E_{x_{0}, \theta_{0}}\left\{x_{\theta_{0}}(\tau(1))^{\gamma} V_{\theta(\tau(1))} x_{\theta_{0}}(\tau(1)) 1_{\{\tau(1) \leq t\}}+x_{\theta_{0}}(t)^{\gamma} V_{\theta_{0}} x_{\theta_{0}}(t) 1_{\{\tau(1)>t\}}-x_{0}^{\prime} V_{\theta_{0}} x_{0}\right\} \\
=\left(\int_{0}^{t} e^{\lambda_{i i} s}\left(\sum_{j \neq i} \lambda_{i j} x_{i}(s)^{\gamma} V_{j} x\{s)\right) d s+x_{i}(t)^{\prime} V_{\imath} x(t) e^{\lambda_{i i} t}-x_{0}^{\prime} V_{i} x_{0}\right) \cdot 1_{\left\{\theta_{0}=i\right\}} \\
=\left(\int _ { 0 } ^ { t } e ^ { \lambda _ { i i } s } \left(\sum_{j \neq i} \lambda_{i j} x_{i}(s)^{\gamma} V_{j} x_{i}(s)+\left(\widehat{A} x_{i}(s)+B_{i} u(s)^{\prime}\right) V_{i} x_{i}(s)+\right.\right. \\
\left.\left.x_{i}(s)^{\gamma} V_{i}\left(\widehat{A}_{i} x_{i}(s)+B \underline{u l}(s)\right)\right) d s\right) \cdot 1_{\left\{\theta_{0}=i\right\}}
\end{gathered}
$$

Therefore, with (3.14) we get that

$$
\begin{aligned}
& E_{x_{0}, \theta_{0}}\left\{\int_{0}^{\tau(1) \wedge t}\left\|C_{\theta(s)} x(s)+D_{\theta(s)} u(s)\right\|^{2} d s+x(\tau(1) \wedge t)^{\gamma} V_{\theta(\tau(1) \wedge t)} x(\tau(1) \wedge t)-x_{0}^{\prime} V_{\theta_{0}} x_{0}\right\}
\end{aligned}
$$

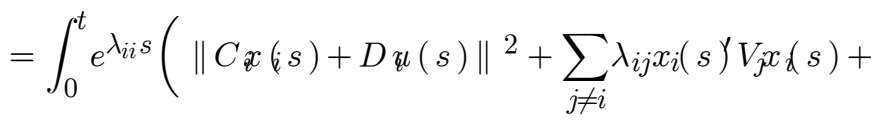

$$
\begin{aligned}
& \left.\left.\left(\widehat{A}_{i} x_{i}(s)+B_{i} u(s)^{\prime}\right) V_{i} x_{i}(s)+x_{i}(s)^{\gamma} V_{i}\left(\widehat{A}_{i} x_{i}(s)+B u \varkappa(s)\right)\right) d s\right) \cdot 1_{\left\{\theta_{0}=i\right\}} \\
& =\int_{0}^{t} e^{\lambda_{i i} s} \varphi_{i}(s)^{\prime}\left(D_{i}^{\prime} D_{i}\right)^{-1} \varphi_{i}(s) d s \cdot 1_{\left\{\theta_{0}=i\right\}}
\end{aligned}
$$

where, $\varphi_{i}(s)=D_{i}^{\prime} D_{i} u(s)+\left(B_{i}^{\prime} V_{i}+D_{i}^{\prime} C\right) x(s)$. Since $V_{\theta_{0}}=R_{\theta_{0}}^{k+1}\left(\mathcal{X}^{0}\right)$, and taking the limit $t \rightarrow \infty$, $V_{\theta(\tau(1) \wedge t)} \rightarrow V_{\theta(\tau(1))}=R_{\theta(\tau(1))}^{k}\left(\mathcal{X}^{0}\right)$ (by definition, $\left.\theta(\tau(1)) \neq i\right)$, we get that 


$$
\begin{aligned}
& E_{x_{0}, \theta_{0}}\left\{\int_{0}^{\tau(1)}\left\|\mathrm{C}_{\theta(s)} x(s)+D_{\theta(s)} u(s)\right\|^{2} d s+\left\|\left(R_{\theta(\tau(1))}^{k}\left(\mathcal{X}^{0}\right)\right)^{1 / 2} x(\tau(1))\right\|^{2}\right\} \\
& =\left(\int_{0}^{\infty} e^{\lambda_{i i} s} \varphi_{i}(s)^{\prime}\left(D_{i}^{\prime} D_{i}\right)^{-1} \varphi_{i}(s) d s+x_{0}^{\prime} R_{i}^{k+1}\left(\mathcal{X}^{0}\right) x_{0}\right) \cdot 1_{\left\{\theta_{0}=i\right\}}
\end{aligned}
$$

and the minimum is achieved when $\varphi_{i}(s)=0,0 \geq s$, for each $i=1, \ldots, N$, showing (3.13).

Similarly, define the following sequence of $\left\{\Im_{t}\right\}$-stopping times

$$
\xi(0):=0, \quad \xi(k):=\min \left\{t>\xi(k-1): \theta(t)>\theta\left(t^{-}\right)\right\}
$$

With this definition, we have a counterpart of Lemma 3.3 for Method I.

Corollary 3.1: For $k=0,1,2, \ldots$, and system (2.3) with a real vector $x_{0}$ and $\theta_{0}=i$,

$$
x_{0}^{\prime} S_{i}^{k}\left(\mathcal{X}^{0}\right) x_{0}=\min E\left(\int_{0}^{\xi(k)}\left\|C_{\theta(s)} x(s)+D_{\theta(s)} u(s)\right\|^{2} d s+\left\|\left(X_{\theta(\xi(k))}^{0}\right)^{1 / 2} x(\xi(k))\right\|^{2}\right)
$$

is verified, where the minimization is over $\{u(t): 0 \leq t \leq \xi(k)\}$.

Proof: Parallels the proof of Lemma 3.1.

It is important to notice that the following holds:

$$
\tau(\eta) \leq \xi(\eta) .
$$

In the sequel, $a$ and $b$ are as in Proposition 2.1 part c), and $c=\max \left\{\left\|\mathcal{X}_{i}^{0}\right\| ; i=1, \ldots, N\right\}$. Denote by $\left(x^{*}(t), u^{*}(t)\right)$ the state and control variables generated by (2.3) when $u^{*}(t)=K_{\theta(t)} x^{*}(t)$ and $\mathcal{P}=\left(P_{1}, \ldots, P_{N}\right), \mathcal{K}=\left(K_{1}, \ldots, K_{N}\right)$ are as is Section 2 and (2.4). Note that

$$
\begin{aligned}
J\left(x_{0}, i\right)=x_{0}^{\prime} P_{i} x_{0} & =E\left(\int_{0}^{\zeta}\left\|\mathrm{C}_{\theta(s)} x^{*}(s)+D_{\theta(s)} u^{*}(s)\right\|^{2} d s+\left\|P_{\theta(\zeta)}^{1 / 2} x^{*}(\zeta)\right\|^{2}\right) \\
& \geq E\left(\int_{0}^{\zeta}\left\|C_{\theta(s)} x^{*}(s)+D_{\theta(s)} u^{*}(s)\right\|^{2} d s\right)
\end{aligned}
$$

holds for any $\left\{\Im_{t}\right\}$ - stopping time $\zeta$.

Lemma 3.4: For $k=0,1,2,, \ldots$, and any real vector $x_{0}$ and $\theta_{0}=i, x_{0}^{\prime} R_{i}^{k}(0) x_{0} \leq x_{0}^{\prime} S_{i}^{k}(0) x_{0}$, and,

$$
\begin{gathered}
x_{0}^{\prime} R_{i}^{k}\left(\mathcal{X}^{0}\right) x_{0} \leq x_{0}^{\prime} S_{i}^{k}\left(\mathcal{X}^{0}\right) x_{0} \\
x_{0}^{\prime} R_{i}^{k}(0) x_{0} \leq x_{0}^{\prime} R_{i}^{k}\left(\mathcal{X}^{0}\right) x_{0} \leq x_{0}^{\prime} P_{i} x_{0}+a c E\left(\mathrm{e}^{\mathrm{b} \tau(k)}\right) \\
x_{0}^{\prime} S_{i}^{k}(0) x_{0} \leq x_{0}^{\prime} S_{i}^{k}\left(\mathcal{X}^{0}\right) x_{0} \leq x_{0}^{\prime} P_{i} x_{0}+a c E\left(\mathrm{e}^{\mathrm{b} \xi(k)}\right) .
\end{gathered}
$$

Moreover, we have that $R_{i}^{k}\left(\mathcal{X}^{0}\right) \rightarrow P_{i}$ and $S_{i}^{k}\left(\mathcal{X}^{0}\right) \rightarrow P_{i}$ as $k \rightarrow \infty$. 
Proof: Relation (3.17) is immediate from Lemma 3.1 (i). Let us show (3.18) only, the other inequalities follow in a similar way. From Lemma 3.1, (3.16), and Proposition 2.1 (c):

$$
\begin{aligned}
x_{0}^{\prime} R_{i}^{k}(0) x_{0} & =\min E\left(\int_{0}^{\tau(k)}\left\|C_{\theta(t)} x(t)+D_{\theta(t)} u(t)\right\|^{2} d t\right) \\
& \leq \min E\left(\int_{0}^{\tau(k)}\left\|C_{\theta(t)} x(t)+D_{\theta(t)} u(t)\right\|^{2} d t+\left\|F_{\theta(\tau(k))}^{1 / 2} x(\tau(k))\right\|^{2}\right) \\
& \left.=x_{0}^{\prime} R_{i}^{k}\left(\mathcal{X}^{0}\right) x_{0} \leq x_{0}^{\prime} P_{i} x_{0}+c E\left(\| x^{*}(\leftarrow k)\right) \|^{2}\right) \leq x_{0}^{\prime} P_{i} x_{0}+a c E\left(\mathrm{e}^{b \tau(k)}\right) .
\end{aligned}
$$

From (3.15), it follows that $0 \leq E\left(\mathrm{e}^{b \xi(k)}\right) \leq E\left(\mathrm{e}^{b \tau(k)}\right) \rightarrow 0$ as $k \rightarrow \infty$ since $\mathrm{e}^{b \tau(k)} \rightarrow 0 \mathbb{P}$-a.s. as $k \rightarrow \infty$. From Theorem 3.1, $R_{i}^{k}(0) \rightarrow P_{i}$ and $S_{i}^{k}(0) \rightarrow P_{i}$ as $k \rightarrow \infty$, and thus, (3.18) and (3.19) leads to $R_{i}^{k}\left(\mathcal{X}^{0}\right) \rightarrow P_{i}$ and $S_{i}^{k}\left(\mathcal{X}^{0}\right) \rightarrow P_{i}$ as $k \rightarrow \infty$.

Proof of Theorem 3.1: It follows from Lemma 3.4.

\section{Proof of Theorem 3.2:}

(i) In view of (3.15), (3.17), (3.18), (3.19) and Lemma 3.4, Method II should have a slower rate of convergence than Method I.

(ii) It follows from Lemma 3.2.

Proof of Theorem 3.3: For Method II one can conclude from Lemma 3.3 that for arbitrary $x_{0}$ and $\theta_{0}$,

$$
\begin{aligned}
J^{k}\left(x_{0}, \theta_{0}\right) & =x_{0}^{l} R_{\theta_{0}}^{k}\left(\mathcal{X}^{0}\right) x_{0} \\
& \leq E_{x_{0}, \theta_{0}}\left\{\int_{0}^{\tau(k)}\left\|C_{\theta(t)} x(t)+D_{\theta(t)} u(t)\right\|^{2} d t+\left\|F_{\theta(\tau(k))}^{1 / 2} x(\tau(k))\right\|^{2}\right\}
\end{aligned}
$$

for any $t \rightarrow u(t)$; in particular for any $u(t)=K_{\theta(t)} x(t)$. Now, suppose that $R_{i}^{k}\left(\mathcal{X}^{0}\right)$ diverges for some $i$, as $k \rightarrow \infty$; it implies that the right-hand side of (3.20) diverges with $\theta_{0}=i, x_{0}$ real vector, and any $t \rightarrow u(t)$, thus, the system can not be mean square stabilizable. Conversely, suppose that $R_{i}^{k}\left(\mathcal{X}^{0}\right) \rightarrow P_{i}$ as $k \rightarrow \infty$, for each $i$. Then $\mathcal{P}$ satisfies (2.1) and we set $K_{i}$ as in (2.4) to conclude for any $x_{0}$ and $\theta_{0}$ that $E_{x_{0}, \theta_{0}}\left\{\int_{0}^{\infty}\left\|\left(C_{\theta(t)}+D_{\theta(t)} K_{\theta(t)}\right) x(t)\right\|^{2} d t\right\}=x_{0}^{\prime} P_{\theta_{0}} x_{0}<\infty$. Since condition H1) or H2) in section 2.2 is satisfied, it follows that $E_{x_{0}, \theta_{0}}\|x(t)\|^{2} \rightarrow 0$, i.e. the system is mean square stable. The proof for Method I follows along the same lines.

\section{Numerical Examples}

\subsection{Examples}


This section presents some numerical tests with the proposed methods, and it includes comparisons with existing methods. Methods I, II, III and IV are easily implemented with cyclical updates using standard Matlab routines for solving Riccati and Lyapunov equations ${ }^{3}$. The examples are the following.

- Example $1(N=3)$ Borrow from [1].

$$
\begin{gathered}
A_{1}=\left(\begin{array}{ccc}
-2.5 & .3 & .8 \\
1 & -3 & .2 \\
0 & .5 & -2
\end{array}\right), B_{1}=\operatorname{diag}\{.707,1,1\}, C_{1}=\left(\begin{array}{c}
\operatorname{diag}\{5,1,3.31\} \\
03 \times 3
\end{array}\right) \\
A_{2}=\left(\begin{array}{ccc}
-2.5 & 1.2 & .3 \\
-.5 & 5 & -1 \\
.25 & 1.2 & -2
\end{array}\right), B_{2}=\operatorname{diag}\{.707,1, .707\}, C_{2}=\left(\begin{array}{c}
\operatorname{diag}\{6.08,8.36,5.83\} \\
03 \times 3
\end{array}\right) \\
A_{3}=\left(\begin{array}{ccc}
2 & 1.5 & -.4 \\
2.2 & 3 & .7 \\
1.1 & .9 & -2
\end{array}\right), B_{3}=\operatorname{diag}\{.707,1,1\}, C_{3}=\left(\begin{array}{c}
\operatorname{diag}\{3.16,4,4.58\} \\
03 \times 3
\end{array}\right) \\
D_{i}=\left(\begin{array}{c}
03 \times 3 \\
I_{3}
\end{array}\right), i=1,2,3 ; \quad \Lambda=\left(\begin{array}{ccc}
-3 & .5 & 2.5 \\
1 & -2 & 1 \\
.7 & .3 & -1
\end{array}\right) .
\end{gathered}
$$

- Example $2(N=6)$ It was produced by augmentation of Example 1 with three more forms, generated randomly. The extra forms and the transition matrix are as follows:

$$
\begin{aligned}
& A_{4}=\left(\begin{array}{lll}
2.7 & .03 & 1.8 \\
.54 & 3.0 & .93 \\
.39 & .89 & 2.3
\end{array}\right), B_{1}=\operatorname{diag}\{.151, .854, .822\}, C_{4}=\left(\begin{array}{c}
\operatorname{diag}\{.988, .334, .760\} \\
03 \times 3
\end{array}\right) \\
& A_{5}=\left(\begin{array}{lll}
-.69 & -1.3 & -1.4 \\
-1.0 & -.67 & -.76 \\
-.40 & -.04 & -.66
\end{array}\right), B_{1}=\operatorname{diag}\{.645, .289, .309\}, C_{5}=\left(\begin{array}{c}
\operatorname{diag}\{.530, .783, .794\} \\
03 \times 3
\end{array}\right) \\
& A_{6}=\left(\begin{array}{lll}
2.0 & .91 & 2.0 \\
2.1 & 1.1 & .90 \\
1.3 & .58 & 2.1
\end{array}\right), B_{1}=\operatorname{diag}\{.838, .546, .795\}, C_{6}=\left(\begin{array}{c}
\operatorname{diag}\{.059, .305, .971\} \\
03 \times 3
\end{array}\right)
\end{aligned}
$$

${ }^{3}$ We employed in the tests the Matlab 5.1 and routines lqr and lyap of the Matlab Control Toolbox, versions revised on 7-18-90. 


$$
\Lambda=\left(\begin{array}{cccccc}
-3 & .5 & .5 & 0 & 1 & 1 \\
1 & -2 & 0 & 1 & 0 & 0 \\
.1 & .2 & -1 & .5 & .1 & .1 \\
0 & 1 & 1 & -3 & .5 & .5 \\
1 & 0 & 0 & 1 & -2 & 0 \\
.5 & .1 & .1 & .1 & .2 & -1
\end{array}\right)
$$

and $D_{i} i=1, \ldots, 6$ as in example 1 .

\subsection{Comparison between Methods I, II, III and IV}

Since the Lyapunov methods (III and IV) require an initialization procedure, we tried three types of comparisons:

- $\mathcal{X}^{0}=0$ for the Riccati methods, and account for time spent in the initialization step L1 for the Lyapunov methods. The initialization matrices $\mathcal{X}^{0}, \mathcal{F}^{0}$ were obtained by solving $N$ uncoupled Riccati equations in $X_{i}^{0}$, with matrices $\mathcal{C}$ and $\mathcal{D}$, and $F_{i}^{0}=-\left(D_{i}^{\prime} D_{i}\right)^{-1}\left(B_{i}^{\prime} X_{i}^{0}+D_{i}^{\prime} C_{i}\right)$. The results for the two examples are shown in Table 4.1.

Table 4.1 Overall Load. (relative precision $\approx 10^{-8}$ )

\begin{tabular}{|c|c|l|c|c|}
\hline & \multicolumn{2}{|c|}{ Example 1 } & \multicolumn{2}{c|}{ Example 2 } \\
\hline & Iterations $^{\dagger}$ & Cpu Time $^{\ddagger}$ & Iterations & Cpu Time \\
\hline Method I & 8 & $0.59(1: 1)$ & 12 & $2.05(1: 1)$ \\
\hline Method II & 13 & $0.98(1: 1.66)$ & 23 & $3.86(1: 1.88)$ \\
\hline Method III & 8 & $0.81(1: 1.37)$ & 15 & $3.02(1: 1.47)$ \\
\hline Method IV & 14 & $1.24(1: 2.10)$ & 21 & $4.02(1: 1.96)$ \\
\hline
\end{tabular}

Note: The fastest method was Method I in the two examples. It spent a cpu time between 1.66 and 2.10 times smaller in the two examples to attain the same precision, if compared with existent methods (Methods II and IV).

- All four methods are initialized with a set of matrices $\mathcal{X}^{0}=\left(X_{1}^{0}, \ldots X_{N}^{0}\right), \mathcal{F}^{0}=\left(F_{1}^{0}, \ldots X_{N}^{0}\right)$ that stabilizes each mode $i$, obtained as described in the previous test. Notice that $\mathcal{F}$ does not satisfy a coupled Lyapunov equation. The time spent with the initialization is not accounted in the Table 4.2.

Table $4.2-\mathcal{X}^{0}$ as in the step L1. (relative precision $\approx 10^{-8}$ )

\begin{tabular}{|c|c|c|c|c|}
\hline & \multicolumn{2}{|c|}{ Example 1 } & \multicolumn{2}{c|}{ Example 2 } \\
\hline & Iterations & Cpu Time & Iterations & Cpu Time \\
\hline Method I & 7 & $0.56(1: 1.05)$ & 12 & $2.11(1: 1)$ \\
\hline Method II & 12 & $0.92(1: 1.73)$ & 22 & $3.72(1: 1.76)$ \\
\hline Method III & 7 & $0.53(1: 1)$ & 14 & $2.47(1: 1.17)$ \\
\hline Method IV & 13 & $0.95(1: 1.79)$ & 20 & $3.56(1: 1.69)$ \\
\hline
\end{tabular}

Note: Method III was slightly faster than Method I in Example 1 whereas method I was the fastest in the Example 2. The time ratios does not vary much in the two examples.

\footnotetext{
${ }^{\dagger}$ The initialization step for the Lyapunov methods is counted as one in the number of iterations.

${ }^{\ddagger}$ In seconds and the relative time ratios among the methods.
} 
- The methods are initialized with a set of matrices that satisfies a coupled Lyapunov equation. The stabilizing control is obtained as described in the first test, and the corresponding coupled equation is obtained using the method in [3]. The time spent in this initialization is not accounted for in Table 4.3. Theorem 3.2 (iii) applies here, concerning the number of iterations.

Table $4.3-\mathcal{X}^{0}$ solution of coupled Lyapunov equation (relative precision $\approx 10^{-8}$ )

\begin{tabular}{|c|c|c|c|c|}
\hline & \multicolumn{2}{|c|}{ Example 1 } & \multicolumn{2}{c|}{ Example 2 } \\
\hline & Iterations & Cpu Time & Iterations & Cpu Time \\
\hline Method I & 6 & $0.48(1: 1.09)$ & 12 & $2.06(1: 1)$ \\
\hline Method II & 11 & $0.86(1: 1.95)$ & 23 & $3.85(1: 1.87)$ \\
\hline Method III & 6 & $0.44(1: 1)$ & 15 & $2.62(1: 1.27)$ \\
\hline Method IV & 11 & $0.78(1: 1.77)$ & 25 & $4.34(1: 2.11)$ \\
\hline
\end{tabular}

Note: Method III was slightly faster than Method I in Example 1 whereas method I was the fastest in the Example 2. We observed that the sequence are monotone decreasing and the number of iterations for the Riccati methods is smaller or equal the number of iterations for the corresponding Lyapunov method, as expected.

The tests run in a Sun 5 Sparc Station, and the cpu time correspond to an average of 6 replications. The residuals of the solutions was monitored by the relative precision criteria: $\max \left\{\left\|\operatorname{Xerr}_{i} / \mathrm{IX}_{i}\right\| \infty\right.$ : $i=1, \ldots, N\}$, where $\|A\|_{\infty}=\max \left\{\left|a_{k j}\right|\right\}$ for a matrix $A$, and ./ indicates the element by element division (array division). The value $\mathrm{Xerr}_{i}$ is produced with the use of the Matlab routine ric.

\subsection{Comparison between the LMI and Methods I and III.}

The convex method was implemented with the package LMIsol [14], dedicated to numerical solution of LMI's. We compare the cpu time with the Matlab routines in section 4.1, adjusted to attain a comparable relative precision. The procedure for Methods I and III and the time accounted is the same as described in the first test of section 4.1 .

Table 4.4 - Comparison with LMI method

\begin{tabular}{|c|c|c|c|c|}
\hline & \multicolumn{3}{|c|}{ Example 1 $\left(\right.$ precision $\left.\approx 10^{-6}\right)$} & \multicolumn{2}{c|}{ Example 2 $\left(\right.$ precision $\left.\approx 10^{-5}\right)$} \\
\hline & Iterations & Cpu Time & Iterations & Cpu Time \\
\hline Method I & 6 & $0.56(1: 1)$ & 9 & $1.55(1: 1)$ \\
\hline Method III & 7 & $0.72(1: 1.29)$ & 12 & $2.47(1: 1.59)$ \\
\hline LMI & 17 & $2.10(1: 3.75)$ & 20 & $8.85(1: 5.71)$ \\
\hline
\end{tabular}

Note: Method I attains the best performance; the performance marks are wider apart when the dimension of the problem increases.

\section{Conclusions}

Tables 4.1, 4.2 and 4.3 indicate a significant gain in the cpu time regarding the forms of carrying new updates: Methods I attains a reduction between 1:1.66 to 1:1.88 when compared with Method II, and Method III attains a reduction between 1:1.33 and 1:1.77 when compared with Method IV. In the best case, the reduction in time is 1:2.10 and in the worse is 1:1.33, see Table 4.1. 
The number of iterations of the Lyapunov methods is always large or equal the number of iterations of the Riccati methods and this is the prevailing factor in the examples. Even if the solution of a Riccati equation is more costly than that of a comparable Lyapunov equation, the best performance is attained by Method I, in most cases, and by Method III in some cases. Most importantly, it performs better in all cases in Tables 4.1 and 4.4 where the overall time spent by the Methods I and III is expressed; the Lyapunov method III spent between $29 \%$ to $59 \%$ more time than the Riccati method I in these two tables.

A second aspect in favor of the Riccati method is the initialization step (L1) required by the Lyapunov method, not needed for the Riccati technique. Moreover the convergence of the Riccati methods is by itself a verification of the mean square stabilizability of the MJLS, as established by Theorem 3.3. 
Appendix: Proof of Proposition 2.3

Consider the ARE in (2.5), and set $\bar{C}=\left(I-D\left(D^{\prime} D\right)^{-1} D^{\prime}\right) C$

$$
\begin{aligned}
& \tilde{L}=\left(\begin{array}{c}
C \\
Y^{1 / 2}
\end{array}\right), \quad \tilde{D}=\left(\begin{array}{c}
D \\
0
\end{array}\right), \quad \tilde{A}=\widehat{A-B}\left(\tilde{D}^{\prime} \tilde{D}\right)^{-1} \tilde{D}^{\prime} \tilde{L}=\widehat{A-B}\left(D^{\prime} D\right)^{-1} D^{\prime} C, \\
& \tilde{C}=\left(I-\tilde{D}\left(\tilde{D}^{\prime} \tilde{D}\right)^{-1} \tilde{D}^{\prime}\right) \tilde{L}=\left(\begin{array}{c}
\bar{C} \\
Y^{1 / 2}
\end{array}\right)
\end{aligned}
$$

Lemma A.1: Suppose that $(\bar{C}, \tilde{A})$ is detectable. Then $(\tilde{C}, \tilde{A})$ is also detectable.

Proof: Obvious.

Lemma A.2: Suppose that $(\widehat{A}, B)$ is stabilizable and $(\bar{C}, \tilde{A})$ is detectable. Then there exists a unique positive semi definite solution $X$ for equation (2.5). Moreover, $\widehat{A}+B K$ is stable, where

$$
K=-\left(D^{\prime} D\right)^{-1}\left(B^{\prime} X+D^{\prime} C\right)
$$

Proof: From Lemma A.1, $(\tilde{C}, \tilde{A})$ is detectable. Notice that equation (2.5) can be written as

$$
\widehat{A}^{\prime} X+X \widehat{A}+\tilde{L^{\prime}} \tilde{L}-\left(X B+\tilde{L^{\prime}} \tilde{D}\right)\left(\tilde{D}^{\prime} \tilde{D}\right)^{-1}\left(B^{\prime} X+\tilde{D}^{\prime} \tilde{L}\right)=0
$$

and the Lemma follows from standard results on ARE's.

Lemma A.3: Suppose that $(\widehat{A}, B)$ is stabilizable and $(\bar{C}, \widetilde{A})$ is detectable, and $X \geq 0$ satisfies:

$$
\widehat{A}^{\prime} X+X \widehat{A}+C C-(X B+C D)\left(D^{\prime} D\right)^{-1}\left(B^{\prime} X+D^{\prime} C\right) \leq 0
$$

Then $\widehat{A}+B K$ is stable with $K$ as in (A.1).

Proof: We can rewrite the above equation as in (2.5) for some appropriate $Y \geq 0$. Therefore considering this $Y \geq 0$ fixed and solving the corresponding ARE we must have that the solution is $X$ by uniqueness of the positive semi definite solution of (2.5). The result follows from Lemma A.2.

\section{Proof of Proposition 2.3:}

First, we prove part (ii). From (2.6) we have

$$
(\widehat{A}+B K)^{y} \tilde{X}+\tilde{X}(\widehat{A}+B K)=-\left(\tilde{Y}+\bar{C}^{\prime} \bar{C}+S B\left(D^{\prime} D\right)^{-1} B^{\prime} S\right) \leq 0
$$

and thus, $\tilde{X} \geq 0$ since $(\widehat{A}+B K)$ is stable. In addition, after some algebraic manipulations, (2.6) can be written as

$$
\begin{aligned}
& \widehat{A}^{\prime} \tilde{X}+\tilde{X} \widehat{A}+C C-(\tilde{X B}+C D)\left(D^{\prime} D\right)^{-1}\left(B^{\prime} \tilde{X}+D C\right) \\
& =-\tilde{Y}-(\tilde{K}-K)(D D)(\tilde{K}-K) \leq 0
\end{aligned}
$$

where $\tilde{K}=-\left(D^{\prime} D\right)^{-1}\left(B^{\prime} \tilde{X}+D^{\prime} C\right)$. Since $(\widehat{A}, B)$ is stabilizable and $(\bar{C}, \tilde{A})$ is detectable, we can apply Lemma A.3 to (A.2) to conclude that $(\widehat{A}+B \tilde{K})$ with $\tilde{K}=-\left(D^{\prime} D\right)^{-1}\left(B^{\prime} \tilde{X}+D^{\prime} C\right)$ is stable. To complete the proof of (ii), we notice that from (A.2) 


$$
\begin{aligned}
& (\widehat{A}+B \tilde{K})^{\prime} \tilde{X}+\tilde{X}(\widehat{A}+B \tilde{K})+(C+D \tilde{K})^{\prime}(C+D \tilde{K}) \\
& +\tilde{Y}+(\tilde{K}-K)\left(D^{\prime} D\right)(\tilde{K}-K)=0
\end{aligned}
$$

and

$$
(\widehat{A}+B \tilde{K}) \bar{X}+\bar{X}(\widehat{A}+B \tilde{K})+\left(C+D \tilde{K}^{\prime}\right)(C+\tilde{D K}) \overline{+} Y=0
$$

Therefore

$$
\begin{aligned}
& (\widehat{A}+B \tilde{K})(\tilde{X}-\bar{X})+(\tilde{X}-\bar{X})(\widehat{A}+B \tilde{K})+(\tilde{Y}-\bar{Y})+ \\
& (\tilde{K}-K)(D D)(\tilde{K}-K)=0 .
\end{aligned}
$$

(A.3) and the stability of $A+B \tilde{K}$ yield that $\bar{X} \leq \tilde{X}$, which completes the proof of (ii).

Part (i): The first assertion in part (i) is proven in Lemma A.2. Let us consider $Y=\bar{Y} \leq \tilde{Y}$ in (2.5). If $(\widehat{A}, B)$ is stabilizable and $(\bar{C}, \tilde{A})$ is detectable, Lemma A.2 states the existence of a unique positive definite solution $\bar{X}$ to the equation (2.5), and it is such that $(\widehat{A}+B \bar{K})$ is stable, with $\bar{K}$ as in (A.1), with $X=\bar{X}$. If we now set $Y=\tilde{Y}$ we can conclude similarly the existence of a unique semi-positive solution $\tilde{X}$ of (2.5) and a $\tilde{K}$ given by (A.1) with $X=\tilde{X}$. After algebraic manipulations similar to that employed in (A.2), (A.3), we get that

$$
\begin{aligned}
& (\widehat{A}+B \tilde{K})(\tilde{X}-\bar{X})+(\tilde{X}-\tilde{X}) \widehat{(A}+\tilde{B} K)+\tilde{(} Y-Y)+ \\
& (\bar{K}-\tilde{K})^{\prime} D^{\prime} D(\bar{K}-\tilde{K})=0 .
\end{aligned}
$$

It then follows from the stability of $A+B \tilde{K}$ that $\bar{X} \leq \tilde{X}$. 


\section{References}

[1] H. Abou-Kandil, G. Freiling and G. Jank, Solution and asymptotic behavior of coupled Riccati equations in jump linear systems, IEEE Trans. Automatic Control 39, 1994, 1631-1636.

[2] M. Ait-Rami and L. E. Ghaoui, LMI optimization for nonstandard Riccati equations arising in stochastic control, IEEE Trans. Automat. Control 41 (11), 1996, 1666-1671.

[3] I. Borno, Parallel computation of the solutions of coupled algebraic Lyapunov equations, Automatica 31 (9), 1995, 1345-1347.

[4] O.L.V. Costa, J.B.R. do Val and J.C. Geromel, A convex programming approach to $\mathcal{H}_{2}$-control of discrete-time Markovian jump linear systems, Int. J. Control 66 (4), 1997, 557-579.

[5] M.H.A. Davis, Markov Models and Optimization, Chapman and Hall, London, 1993.

[6] J.B.R. do Val, J.C. Geromel and O.L.V. Costa, Uncoupled Riccati iterations for the linear quadratic control problem of discrete-time Markov jump linear systems, IEEE Trans. Automat. Control, to appear.

[7] M.D. Fragoso, O.L.V. Costa, and C.E. de Souza, A new approach to linearly perturbed Riccati equations arising in stochastic control, Applied Mathematics and Optimization 37, 1998, 99-126.

[8] X. Feng, K.A. Loparo, Y. Ji and H.J. Chizeck, Stochastic stability properties of jump linear systems, IEEE Trans. Automat. Control 37 (1), 1992, 38-53.

[9] Z. Gajic and I. Borno, Lyapunov iterations for optimal control of jump linear systems at steady state, IEEE Trans. Automat. Control 40 (11), 1995, 1971-1075.

[10] Z. Gajic and R. Losada, Monotonicity of algebraic Lyapunov iterations for optimal control of jump parameter linear systems, preprint, Rutgers University, 1997.

[11] Y. Ji and H.J. Chizeck, Controllability, observability and continuous-time Markovian jump linear quadratic control, IEEE Trans. Automat. Control 35 (7), 1990, 777-788.

[12] D.L. Kleinman, On a iterative technique for Riccati equation computations, IEEE Trans. Automat. Control 13, 1968, 114-115.

[13] M. Mariton, Jump linear systems in automatic control, Marcel Dekker, New York, 1990.

[14] M. C. Oliveira, D.P. Faria and J.C. Geromel, LMIsol user's guide. Available at http:// dt.fee.unicamp.br/ carvalho/\#soft

[15] W. Wonham, On a matrix Riccati equation of stochastic control, SIAM J. Contr. Optim. 6, 1968, 681-697.

[16] W. Wonham, Random difference equations in control theory, in Probabilistic Methods in Applied Mathematics, A. Bharucha-Reid, ed., Academic Press, New York, 131-212 